\title{
PRMT5 inhibition modulates murine dendritic cells activation by inhibiting the metabolism switch: a new therapeutic target in periodontitis
}

\author{
Wenxiang Mi ${ }^{1,2 \#}$, Shichong Qiao ${ }^{1 \#}$, Xiaomeng Zhang ${ }^{1}$, Dongle Wu ${ }^{1}$, Linyi Zhou ${ }^{1}$, Hongchang Lai $^{1}$ \\ ${ }^{1}$ Department of Implant Dentistry, Shanghai Ninth People's Hospital, College of Stomatology, Shanghai Jiao Tong University School of Medicine; \\ National Clinical Research Center for Oral Diseases; Shanghai Key Laboratory of Stomatology \& Shanghai Research Institute of Stomatology, \\ Shanghai, China; ${ }^{2}$ Laboratory of Oral Microbiota and Systemic Diseases, Shanghai Ninth People's Hospital Research Center, Shanghai Jiao Tong \\ University School of Medicine, Shanghai, China \\ Contributions: (I) Conception and design: W Mi, S Qiao, H Lai; (II) Administrative support: H Lai; (III) Provision of study materials or patients: W \\ Mi, S Qiao, X Zhang; (IV) Collection and assembly of data: W Mi, S Qiao, D Wu; L Zhou; (V) Data analysis and interpretation: W Mi, S Qiao, X \\ Zhang; (VI) Manuscript writing: All authors; (VII) Final approval of manuscript: All authors. \\ "These authors contributed equally to this work. \\ Correspondence to: Prof. Hongchang Lai. Department of Implant Dentistry, Shanghai Ninth People's Hospital, College of Stomatology, Shanghai Jiao \\ Tong University School of Medicine; National Clinical Research Center for Oral Diseases; Shanghai Key Laboratory of Stomatology \& Shanghai \\ Research Institute of Stomatology, 639 Zhizaoju Road, Shanghai 200011, China. Email: lhc9@hotmail.com.
}

Background: Protein arginine methyltransferase 5 (PRMT5) catalyzes the methylation of arginine residues in multiple proteins. Recent reports have highlighted the anti-inflammatory role of PRMT5. Dendritic cells (DCs) are well-known professional antigen-presenting cells that are crucial for immune response initiation. However, whether PRMT5 participates in DC immunity processes is unknown.

Methods: In an in vitro experiment, a PRMT5 inhibitor (EPZ015666) was used to inhibit PRMT5 expression, and lipopolysaccharide (LPS) stimulation was applied to mimic the inflammation context. Proinflammatory cytokine production, interferon-stimulated genes (ISGs), costimulatory molecules, major histocompatibility complex (MHC) expression and DC metabolism were measured following PRMT5 inhibition and LPS stimulation. In an in vivo study, we first tested PRMT5 mRNA and protein expression in a BALB/c mouse ligature-induced periodontitis model. Then, we evaluated changes in periodontal tissue and DC migration to cervical lymph nodes after local treatment with the PRMT5 inhibitor.

Results: The in vitro results revealed that PRMT5 inhibition attenuated DC activation and maturation by inhibiting the expression of proinflammatory cytokines, ISGs, costimulatory molecules, and MHC induced by LPS stimulation. We also found that inhibition of PRMT5 blocked the DC metabolic switch to glycolysis. In the in vivo study, we found that PRMT5 inhibition reversed the severity of the lesions and slowed the migration of DCs to cervical lymph nodes.

Conclusions: The results show a critical role of PRMT5 in the control of DC activation through inhibition of the metabolic switch and indicate that PRMT5 is a promising therapeutic target in periodontitis.

Keywords: Dendritic cells (DCs); inflammation; immunity; metabolism; periodontitis

Submitted Nov 09, 2020. Accepted for publication Mar 05, 2021.

doi: $10.21037 / \mathrm{atm}-20-7362$

View this article at: http://dx.doi.org/10.21037/atm-20-7362 


\section{Introduction}

Dendritic cells (DCs) function as both innate immune cells that detect bacteria and antigen-presenting cells that activate adaptive immunity (1). In peripheral tissues, various pattern-recognition receptors expressed by DCs, such as Toll-like receptors (TLRs), detect microbial infection and activate DCs (2). Activated DCs begin to migrate and become mature, releasing cytokines, chemokines and costimulatory molecules to orchestrate antigen capture, perform processing and deliver antigen-derived peptides to naive $\mathrm{T}$ cells (3). The activation and maturation states of DCs determines the subsequent immune response by regulating different helper $T$ cell responses (4).

Lipopolysaccharide (LPS) from gram-negative bacteria is a TLR4 ligand and DC activator (5). LPS regulates the DC metabolic switch from oxidative phosphorylation (OXPHOS) to glycolysis to maintain sufficient energy for survival and activation (6-8). More specifically, pyruvate enters the mitochondria of resting DCs and is converted to acetyl-CoA. Through the tricarboxylic acid (TCA) cycle and OXPHOS, acetyl-CoA is catalytically converted into large amounts of ATP. However, upon stimulation with LPS, increased glucose consumption and lactate production are observable within a short amount of time, pyruvate is converted to lactate to generate ATP, and glycolysis becomes the major energy-producing pathway to promote DC activation during the immune response (9). Nitric oxide production and AKT are two possible mechanisms that influence the DC metabolic state. Inducible NO synthase (iNOS) expression occurs simultaneously with mitochondrial dysfunction in LPS-stimulated DCs due to NO-mediated inhibition of enzymes involved in mitochondrial electron transport $(8,10)$. By phosphorylating related enzymes, AKT itself participates in the mechanism by which DC metabolism is regulated (11). Downstream/ upstream kinases of AKT also have roles in maintaining the helper $\mathrm{T}$ cell balance, accelerating DC activation, and enhancing the DC antigen presenting function $(4,12)$.

Protein arginine methyltransferase 5 (PRMT5) is a type II enzyme in the PRMT family. PRMT5 is responsible for most symmetrical demethylation events (13) and is involved in diverse cellular processes. In addition to its wellstudied anti-tumor activity (14-17), PRMT5 is among the four PRMTs that are correlated with inflammation (18). PRMT5 has been shown to be upregulated in inflammatory diseases, such as Crohn's disease, rheumatoid arthritis and endothelial cell inflammation, and inhibition of PRMT5 reduces inflammation (19-21). Moreover, PRMT5 promotes $\mathrm{T}$ cell maintenance in vitro $(22,23)$. PRMT5 inhibition protects mice from autoimmune diseases and transplant rejection (23-25), and circ-PRMT5 has been reported to regulate tumor cell metabolism by enhancing glycolysis activity (26). However, whether PRMT5 modulates DCs through metabolism has not yet been studied.

Periodontitis is one of the most common inflammation types and causes bone loss in humans (27). The biological structure of periodontal tissue promotes bacterial colonization, which is detected by DCs in the oral mucosa. The DCs become activated, secreting cytokines and upregulating surface costimulatory molecules. At the same time, DCs migrate to cervical lymph nodes, where the captured pathogen is presented with the help of highly expressed major histocompatibility complex (MHC)-I and MHC-II molecules to initiate immunity activation (28). These events are tightly monitored to ensure that the immune response meets the requirement for pathogen elimination without inducing tissue damage by generating excessive inflammation. Thus, we used a mouse ligatureinduced periodontitis model to test the in vivo effect of PRMT5 inhibition on periodontitis through DCs. The PRMT5 inhibitor EPZ015666 was first identified and characterized in 2015 (29) and has demonstrated its value as a validated chemical probe in the inhibition of rheumatoid arthritis and different tumor types by targeting PRMT5 in animal models (29-31). Here, we chose the BALB/c mouse strain because it is more susceptible to periodontitis than other strains, such as C57BL/6J (32).

Here, our data show that PRMT5 inhibition dampens LPS-stimulated DC activation by attenuating the DC metabolic switch to glycolysis. We also highlight the role of PRMT5 as a promising therapeutic target in periodontitis.

We present the following article in accordance with the ARRIVE reporting checklist (available at http://dx.doi. org/10.21037/atm-20-7362).

\section{Methods}

\section{Culture of murine bone marrow-derived DCs (BMDCs)}

Specific pathogen-free BALB/c mice of both genders were obtained from the Charles River Laboratory (Beijing, China). All mouse experiments were approved by the Animal Care and Experiment Committee of Shanghai Jiao Tong University School of Medicine (approval ID: HKDL [2018]461), in compliance with the National institutional 
guidelines for the care and use of animals. Mice aged 9-11 weeks were used in the experiments. BMDCs were isolated using a previously described protocol (33). Briefly, bone marrow was flushed from the femurs of BALB/c mice. Granulocyte-macrophage colony-stimulating factor (GM-CSF) (R\&D Systems, USA) was added to a final concentration of $20 \mathrm{ng} / \mathrm{mL}$, and the mixture was cultured with RPMI-1640 medium supplemented with $10 \%$ fetal bovine serum (FBS; HyClone, USA) plus 1\% antibiotics (Gibco, USA) in an incubator at $37{ }^{\circ} \mathrm{C}$ and an atmosphere containing $5 \% \mathrm{CO}_{2}$. On day 3 , new medium containing the same GM-CSF concentration was added. On day 6, the cells were harvested by gently pipetting away the nonadherent cells with the culture medium.

\section{Measurement of inflammatory cytokine levels via ELISAs}

Primary BMDCs were stimulated with different concentrations of EPZ015666 (Selleck, TX, USA) in the presence or absence of LPS $(100 \mathrm{ng} / \mathrm{mL})$ (Sigma, MO, USA). The levels of IL-6, TNF- $\alpha$, IL-10, and IL-12p70 in the supernatants of the DCs were measured with ELISA kits (Neobioscience, Shenzhen, China) according to the manufacturer's instructions. Briefly, samples and a reference standard were added to the ELISA plate and then incubated for $90 \mathrm{~min}$ with the plate sealed and covered. A biotinylated detection antibody and HRP conjugate were sequentially added, and the samples were completely washed and incubated after the addition of each reagent. The substrate reagent was then added to each well and incubated with the cells. The stop solution was added to stop the reaction, and the absorbance of each well was measured at $450 \mathrm{~nm}$.

\section{Flow cytometry analysis of DC viability and activation}

Primary BMDCs were harvested and stimulated with different concentrations of EPZ015666 (Selleck) in the presence or absence of LPS (100 ng/mL) (Sigma). After the indicated times, BMDCs were either stained with annexin $\mathrm{V}$ and propidium iodide according to the manufacturer's instructions (BD Pharmingen, Oxford, UK) for the cell viability test or prepared for flow cytometry analysis. Briefly, stimulated BMDCs were collected, washed and resuspended to a density of $2 \times 10^{7}$ cells $/ \mathrm{mL}$. Then, $50 \mu \mathrm{L}$ of cell suspension was distributed into tubes and preincubated with Fc block (BD) for 5 min. Predetermined optimal amounts of anti-mouse Cy5.5-CD11c, PE-Cy7-CD86, APC-CD40, PE-CD80, Alexa647-MHC-II (all BD) and
PE-MHC-I (Biolegend, San Diego, CA, USA) antibodies were added and then incubated with cells for $30 \mathrm{~min}$ on ice. The cell samples were washed twice to remove excess antibody, resuspended in $500 \mu \mathrm{L}$ of staining buffer (all BD), and analyzed with a FACS LSRFortessa flow cytometer (BD Bioscience, CA, USA) within $1 \mathrm{~h}$. FlowJo software (Tree Star, OR, USA) was used to analyze the flow cytometry data.

\section{Western blotting (WB)}

Primary BMDCs were harvested and stimulated with different concentrations of EPZ015666 (Selleck) in the presence or absence of LPS (100 ng/mL) (Sigma). After the indicated times, BMDCs were collected and lysed with RIPA buffer containing protease and phosphatase inhibitors (Beyotime, Shanghai, China). The protein samples $(25 \mathrm{mg}$ ) were separated on 10\% SDS-polyacrylamide gels (Beyotime) before being transferred to PVDF membranes (Beyotime). After blocking with $5 \%$ nonfat milk in TBST for $1 \mathrm{~h}$, the membranes were incubated with primary antibody against PRMT5 (Abcam) (Cell Signaling Technology, MA, USA) overnight at $4{ }^{\circ} \mathrm{C}$. After three washes with TBST on the next day, the membranes were incubated with secondary HRP-labeled anti-rabbit/anti-mouse antibody (Beyotime). Immunoreactivity was visualized using a Fusion Pulse 6 system (Vilber Lourmat, France).

\section{Nitric oxide quantification}

Primary BMDCs were harvested and stimulated with different concentrations of EPZ015666 (Selleck) in the presence or absence of LPS (100 $\mathrm{ng} / \mathrm{mL})$ (Sigma). After the indicated times, cells were collected, washed with PBS, and then lysed with the Buffer for a Nitric Oxide Assay (Beyotime). The reduction of nitrate into nitrite was performed by adding nitrate reductase, according to the instructions provided, with a Total Nitric Oxide Assay Kit (Beyotime). After clearing the extra NADPH with lactate dehydrogenase, NO levels were measured by calculating the nitrite concentration from a standard curve composed of a series of BSA (Beyotime) standards measured at $562 \mathrm{~nm}$.

\section{Assessment of mitochondrial respiration and glycolysis}

Primary BMDCs were plated in XF-24 cell culture plates and stimulated with LPS and/or EPZ015666 for 1 or $24 \mathrm{~h}$. After two washes with XF assay medium, the cells were treated with $2 \mu \mathrm{M}$ oligomycin, $1 \mu \mathrm{M}$ fluoro- 
carbonyl cyanide phenylhydrazone (FCCP), and $0.5 \mu \mathrm{M}$ rotenone plus antimycin-A (Agilent Technologies, CA, USA) for measurement of the oxygen consumption rate (OCR) or $10 \mathrm{mM}$ D-glucose, $2 \mu \mathrm{M}$ oligomycin and $50 \mathrm{mM}$ 2-deoxyglucose (Agilent Technologies) for measurement of the extracellular acidification rate (ECAR). The analyses were performed using a Seahorse XF-24 analyzer (Agilent Technologies).

\section{Ligature-induced periodontitis model}

All mouse experiments were approved by the Animal Care and Experiment Committee of Shanghai Jiao Tong University School of Medicine. Specific pathogen-free BALB/c mice (10 per group) of both genders were obtained from the Charles River Laboratory (Beijing, China), and each cage housed a maximum of 5 mice.

Mice of both genders (aged 9-11 weeks, with a weight range of 20-24 g) were randomly assigned to one of three groups: a control group, ligatured group (Liga) or ligatured plus EPZ015666 (Liga + EPZ) group. On the day of surgery, all mice were anesthetized by intraperitoneal injection, and periodontitis was induced in the Liga and Liga + EPZ groups by tying 5-0 cotton ligatures around both sides of the maxillary second molars using a previously described protocol (34). Mice were fed Ready Jelly (ReadyDietech, Shenzhen, China) on the first day after surgery and then a normal diet for the remainder of the study. For the Liga + EPZ group, EPZ015666 (Selleck, Houston, USA) was purchased and diluted with $2 \%$ DMSO $+30 \%$ PEG300 + normal saline to a final concentration of $1.25 \mu \mathrm{g} / \mu \mathrm{L}$. This concentration was determined in a preliminary experiment (data not shown). EPZ015666 was injected into three sites in the palatal gingiva of the second molar $(40 \mu \mathrm{L} /$ per mice) one day before ligation. Because this step takes a few minutes, we let the mice sleep under respiratory anesthesia with a specialized anesthesia machine (MSS, London, UK). Injections were did done every day until the mice were sacrificed on day 9 .

\section{Polymerase chain reaction}

For the in vivo study, gingival tissue from mice in different groups (8-10 mice per group) was excised from the palatal side of the maxillary molars according to a previously described protocol (35) and disrupted by rapid agitation in the presence of beads in TissueLyser (Jingxin, Shanghai, China). For the in vitro study, primary BMDCs were harvested and stimulated with EPZ015666 (Selleck) in the presence or absence of LPS (100 $\mathrm{ng} / \mathrm{mL}$ ) (Sigma) for the indicated times. Next, mRNA was extracted using a RNeasy Mini Kit (Qiagen, CA, USA) and reverse-transcribed using a PrimeScript ${ }^{\mathrm{TM}}$ RT Reagent Kit (TaKaRa, Otsu, Japan). Real-time PCR was performed using a LightCycler/ LightCycler 480 System (Roche Diagnostics, Switzerland) with SYBR $^{\circledR}$ Premix Ex Taq ${ }^{\text {TM }}$ (TaKaRa, Otsu, Japan). The specific primers were synthesized by Sangon (Sangon, Shanghai, China), and gene expression was normalized to GAPDH mRNA expression (Sequences will be provided on request).

\section{Preparation of histological specimens}

On the sacrifice day, isolated mouse skulls were washed with PBS and fixed with $10 \%$ paraformaldehyde at $4{ }^{\circ} \mathrm{C}$ (Beijing, China). After 24 h, samples were washed twice with PBS and stored in 10\% EDTA (Sangon) for 4-5 weeks for decalcification. When the hard tissues were able to be easily poked with a needle, they were deemed ready for paraffin embedding (Macklin, Shanghai, China).

\section{Hematoxylin and eosin (HE) and histological tartrate- resistant acid phosphatase (TRAP) staining}

Briefly, a sufficient amount of deionized water was prewarmed to $37{ }^{\circ} \mathrm{C}$. The tissue slices were dewaxed in xylene, dehydrated in an ethyl alcohol series, and rinsed thoroughly with deionized water. Then, the slices were either stained with $\mathrm{HE}$ or processed as described below for TRAP staining: a cocktail of the reagents was prepared according to the manufacturer's instructions (SigmaAldrich, MO, USA). The cocktail was warmed to $37^{\circ} \mathrm{C}$ and added to the tissue on the slide in a dropwise manner before incubation for $1 \mathrm{~h}$ in a $37^{\circ} \mathrm{C}$ incubator protected from light. The slides were rinsed with deionized water and counterstained for $2 \mathrm{~min}$ in a hematoxylin solution. The slides were rinsed again with alkaline tap water for several minutes and then evaluated under a microscope.

\section{Immunobistochemistry (IHC)}

An HRP/DAB Detection IHC Kit (Abcam, Cambridge, USA) was used for IHC staining. Briefly, primary rabbit monoclonal antibody against CD11c (Abcam) or PRMT5 (Abcam) and a primary antibody control were applied to lymph node slices or gingiva tissue slices individually and 
incubated overnight at $4{ }^{\circ} \mathrm{C}$, followed by incubation with a biotinylated secondary anti-rabbit antibody (Abcam) for 10 $15 \mathrm{~min}$ at room temperature. After 3-4 washes, the slices were incubated with streptavidin peroxidase to amplify the signal, followed by incubation with the DAB chromogen for final coloration.

\section{Determination of bone resorption}

On the day of sacrifice, isolated mouse skulls were boiled, defleshed and brushed before being stored in hydrogen peroxide for bleaching overnight. The skull was trimmed, and the maxillae were stained with $0.5 \%$ eosin (Sangon) and $1 \%$ methylene blue (Macklin, Shanghai, China). Images of the maxillae were captured using a Leica M165 C microscope (Leica, Germany) equipped with a Leica MC170 camera (Leica). Measurements were performed at two sites on the first molar (distal cusp and distopalatal/distobuccal groove), three sites on the second molar (mesiopalatal/ buccal cusp, palatal/buccal groove, and distopalatal/buccal cusp), and one site on the third molar (palatal/buccal). Bone resorption (10 samples per group) was defined as the total bone loss at 6 sites by measuring the distance between the cementoenamel junction (CEJ) and the alveolar bone crest (ABC). The results are presented in $\mathrm{mm}$.

\section{Statistical analysis}

GraphPad Prism software (version 5) was used for statistical analysis. The results are reported as the means \pm standard errors of the means (means \pm SEM). An unpaired two-tailed Student's $t$-test and paired ratio two-tailed Student's $t$-test were used for comparisons between two groups. One-way ANOVA followed by Bonferroni's multiple comparisons test, two-way ANOVA, and the Sidak multiple comparisons test were used for multiple comparisons. A P value of 0.05 was considered statistically significant.

\section{Results}

\section{PRMTS inbibition reduces DC activation without affecting DC survival}

BMDCs were treated with titrated doses of EPZ015666 in the presence or absence of LPS to measure cell viability. The highest EPZ015666 dose $(640 \mu \mathrm{M})$ killed DCs after 24 h of LPS stimulation (Figure $1 A$ ). Although DC viability did not decrease significantly upon LPS stimulation, we still excluded this dose because of its toxicity. Next, we further evaluated DC viability after 48 and $72 \mathrm{~h}$ and found that doses less than $160 \mu \mathrm{M}$ did not affect DC viability (Figure $1 B, C$ ). We then measured cytokine levels and observed a dosedependent decrease in TNF- $\alpha$ and IL-6 levels (2 times) and an obvious decrease in the IL-12p70 level (2 times) in cells treated with $160 \mu \mathrm{M}$ EPZ015666 at $24 \mathrm{~h}$ poststimulation (Figure 1D,E,F). EPZ015666 tended to induce a dosedependent increase in the IL-10 level (up to 1.3 times), but the difference was not statistically significant (Figure 1G,H). Based on the results, $160 \mu \mathrm{M}$ is the optimal dose that suppresses proinflammatory cytokine production in DCs without compromising cell viability.

\section{PRMT5 inbibition downregulates ISG expression and decreases the expression of MHC and costimulatory molecules in LPS-activated DCs after 24-48 b}

In as little as $1 \mathrm{~h}$, PRMT5 inhibition alone increased the levels of all ISG transcripts. When applied in combination with LPS, the PRMT5 inhibitor decreased Ifnb and Isg15 expression (1.8-2 times) but increased the expression of the other three molecules $(\mathrm{Mx} 1, \operatorname{Irf} 7$ and $\mathrm{Cxcl10})$ (1.5-1.8 times) (Figure 2A,B,C,D,E). At $24 \mathrm{~h}$, the PRMT5 inhibitor alone increased Mx1 and Isg15 expression. When administered with LPS, the PRMT5 inhibitor significantly decreased the expression of all ISGs (2-9 times). Since costimulatory and $\mathrm{MHC}$ molecules are also required for DC activation and maturation, we measured the level of these molecules and observed that treatment with the PRMT5 inhibitor alone decreased the MHC-II level at $24 \mathrm{~h}$ (1.4 times); when administered with LPS, the PRMT5 inhibitor decreased the expression of three costimulatory molecules and MHC-II at $24 \mathrm{~h}$ (1.2-1.5 times). The PRMT5 inhibitor also resulted in stronger inhibition of the expression of the three costimulatory molecules and MHC molecules at $48 \mathrm{~h}$ after LPS stimulation (1.1-1.4 times) (Figure 2F, G,H,I,7). Thus, PRMT5 inhibition decreases LPS-induced DC activation and maturation by deregulating ISGs and the expression of MHC and costimulatory molecules at 24-48 h.

\section{PRMT5 inbibition blocks the LPS-induced DC metabolic switch mainly by attenuating glycolysis}

Because the aforementioned data showed a general inhibitory effect of the PRMT5 inhibitor, we next examined whether PRMT5 inhibition plays a profound role in regulating the metabolism of inflammatory 
A

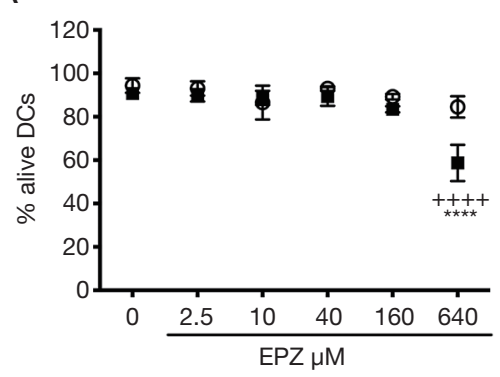

D

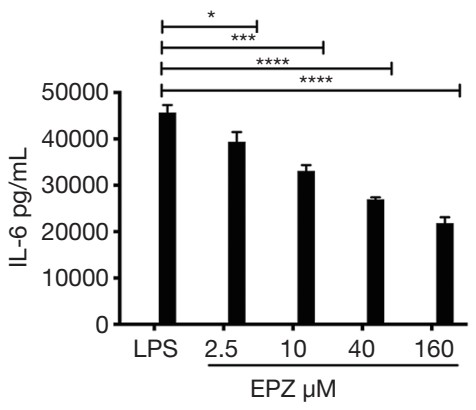

G

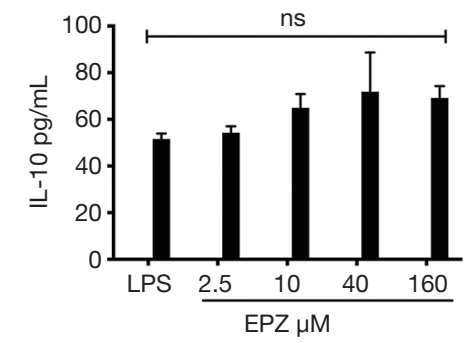

B

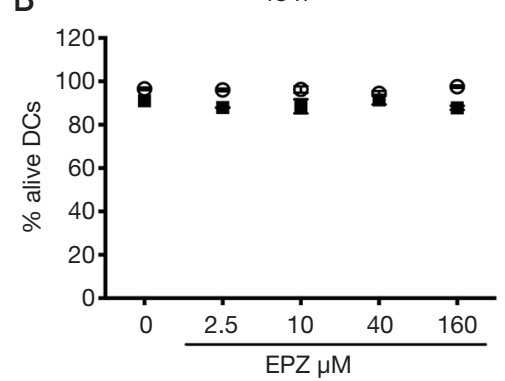

E

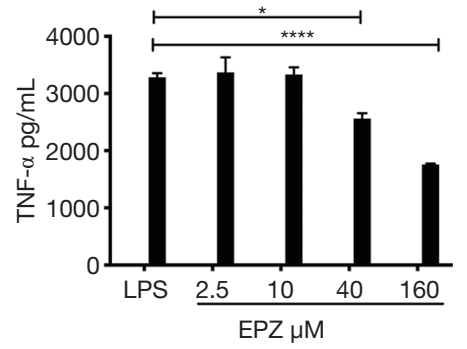

$\mathrm{H}$

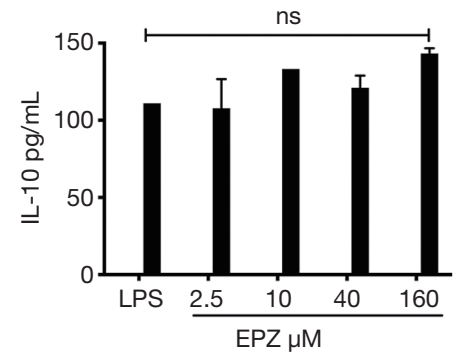

C

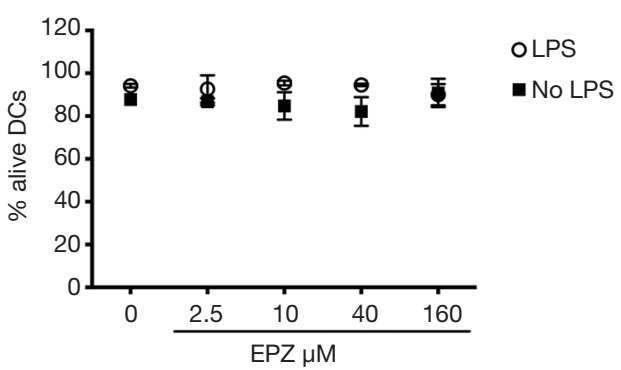

F

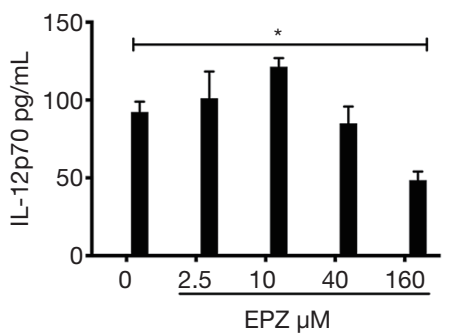

Figure 1 EPZ015666 dose titration response. (A,B,C) DC viability. Flow cytometry analysis of cells stained with PI and annexin V. One-way ANOVA and the Bonferroni multiple comparisons test were used to compare data between the blank control and groups with no LPS (marked as "+"). Two-way ANOVA and the Sidak multiple comparisons test were used to compare data between groups treated with the same EPZ015666 concentration with or without LPS (marked as “*”). (D,E,F,G,H) Inflammatory cytokine production. IL-6, TNF- $\alpha$, IL-12p70 and IL-10 expression levels after LPS stimulation were analyzed via ELISA. One-way ANOVA and the Bonferroni multiple comparisons test were used for data analysis. All data are representative of 3 independent in vitro experiments. ${ }^{*}, \mathrm{P}<0.05 ;{ }^{* * *}, \mathrm{P}<0.001 ;{ }^{* * *}, \mathrm{P}<0.0001$. DC, dendritic cell.

DCs. We performed metabolic assays to obtain detailed measurements of mitochondrial respiration and glycolysis. In the short term, LPS induced increases in the OCR and ECAR, as expected (1.3-1.6 times). However, the PRMT5 inhibitor did not change these rates, regardless of whether it was applied alone or with LPS, and it significantly decreased the maximal mitochondrial respiration activity (1.4 times) (Figure $3 A, B, C$ ). After $24 \mathrm{~h}$ of stimulation, the LPS-induced increase in the OCR was substantially decreased (5 times), although the ECAR remained high. Treatment with the PRMT5 inhibitor alone significantly decreased the ECAR (1.4 times) and blocked the ECAR even more obviously when the inhibitor was applied with LPS stimulation (1.8 times). Again, the PRMT5 inhibitor did not affect the OCR but significantly decreased the maximal mitochondrial respiration activity (1.6 times) (Figure 3D,E,F,G,H). We measured iNOS expression to explore the molecular mechanism. The PRMT5 inhibitor reversed the iNOS 

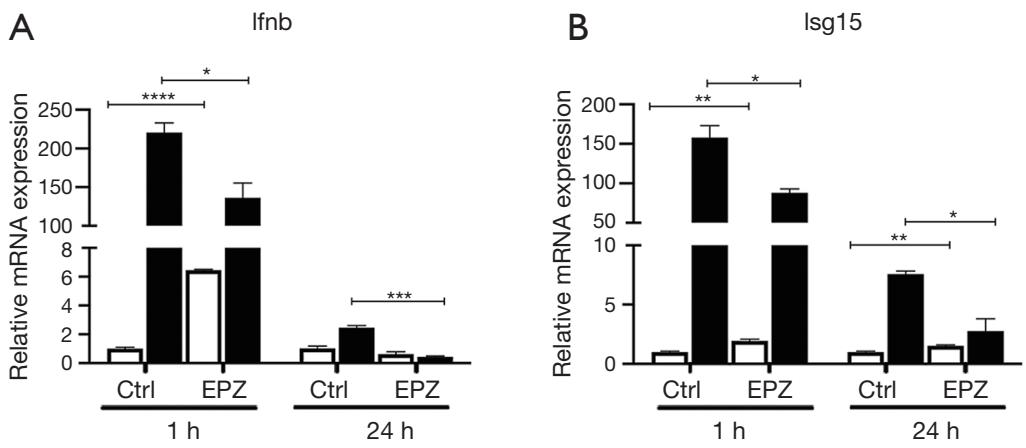

C IIf7
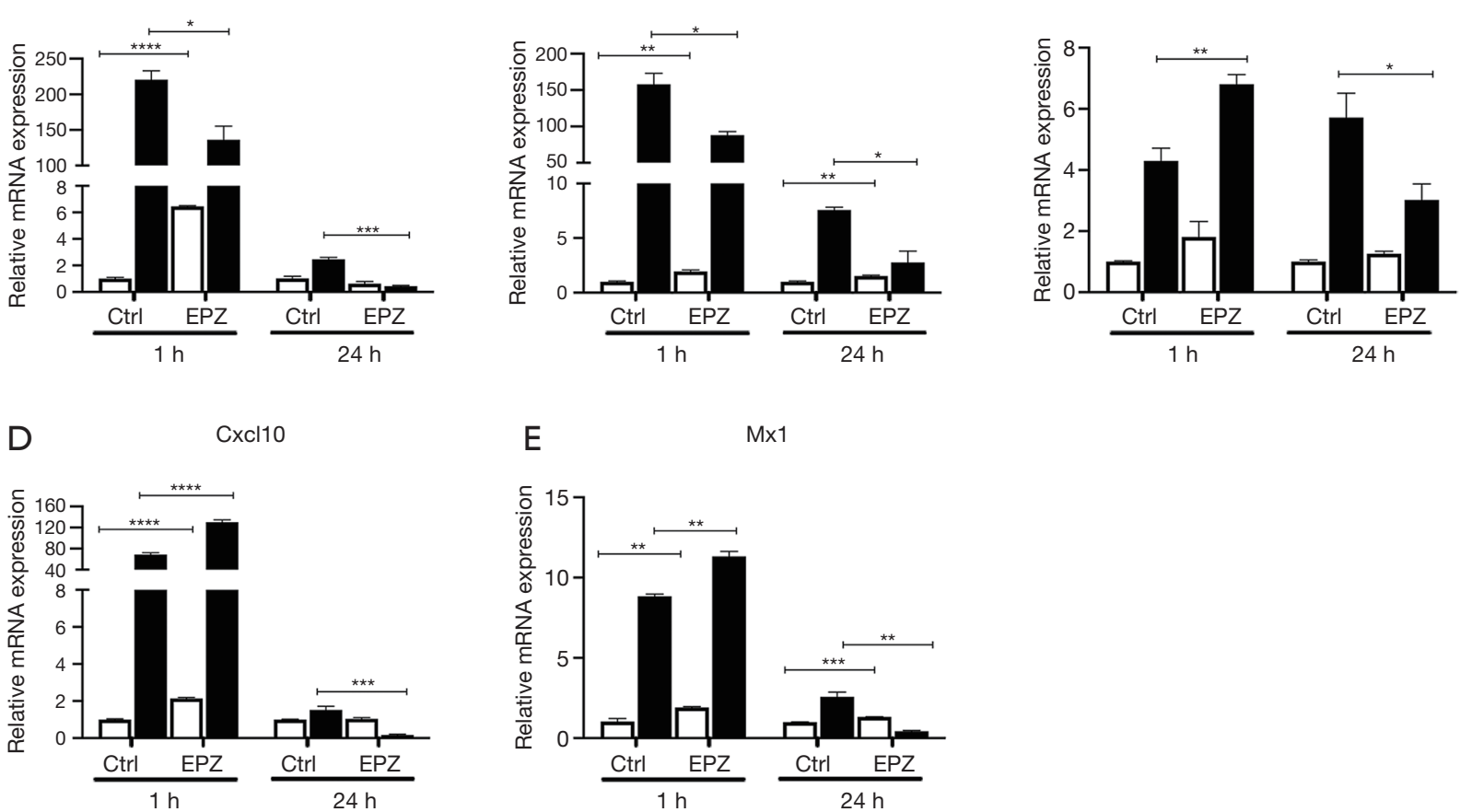

$\square$ No LPS LPS

F
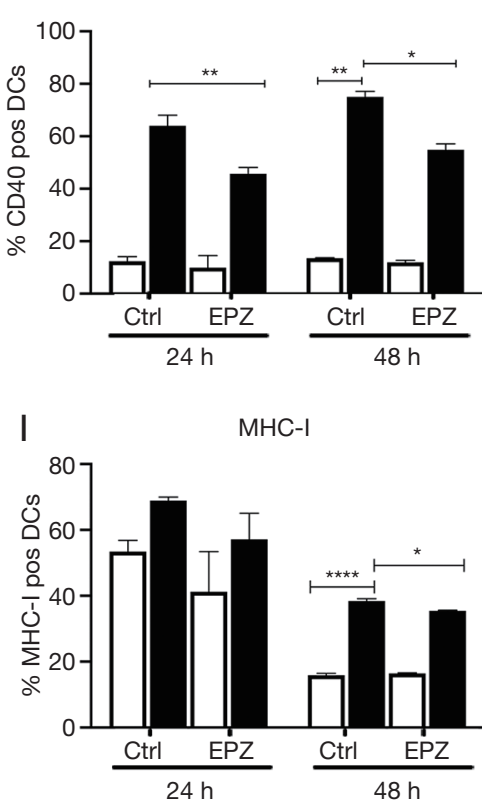

E

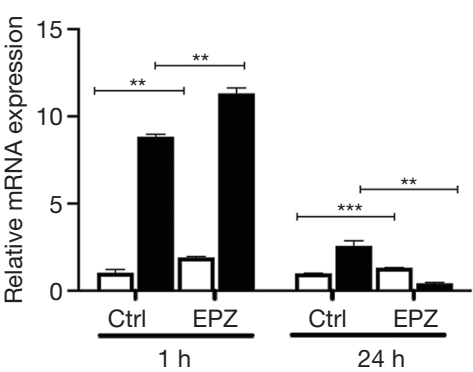

G

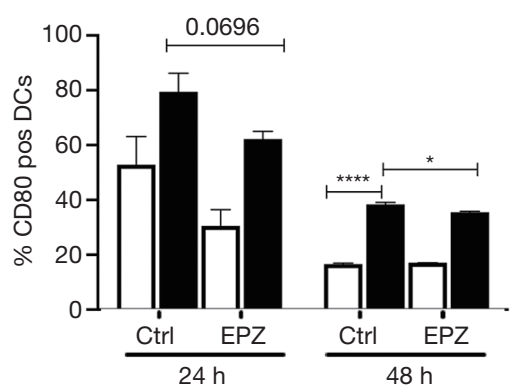

MHC-II

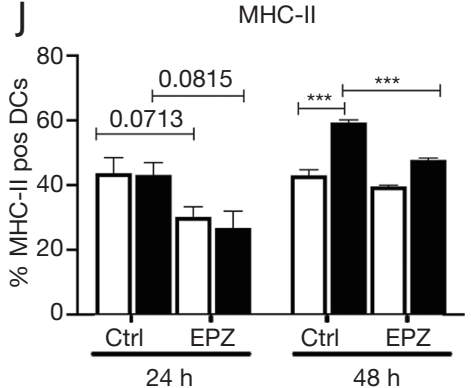

$\mathrm{H} \quad \mathrm{CD} 86$

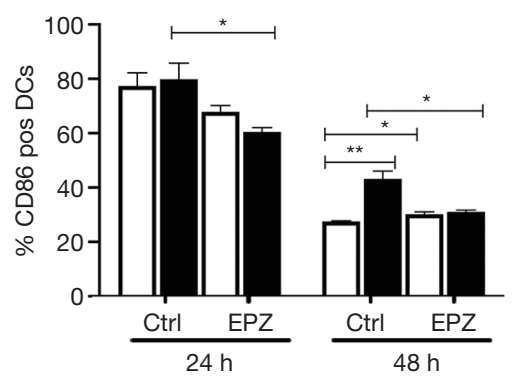

Figure 2 PRMT5 inhibition regulates DC activation and maturation under LPS stimulation. (A,B,C,D,E) Quantitative RT-PCR analysis of interferon-stimulated gene (ISG) expression after 1 or $24 \mathrm{~h}$ of PRMT5 inhibition. (F,G,H,I,J) Flow cytometry analyses of the three surface costimulatory molecules and MHC-I and MHC-II after 24 or 48 h of PRMT5 inhibition. Unpaired two-tailed Student's $t$-test was used for data analysis. *, $\mathrm{P}<0.05$; **, $\mathrm{P}<0.01$; ${ }^{* * *}, \mathrm{P}<0.001$; ${ }^{* * *}, \mathrm{P}<0.0001$. All data are representative of 3 independent in vitro experiments. DC, dendritic cell; MHC, major histocompatibility complex. 

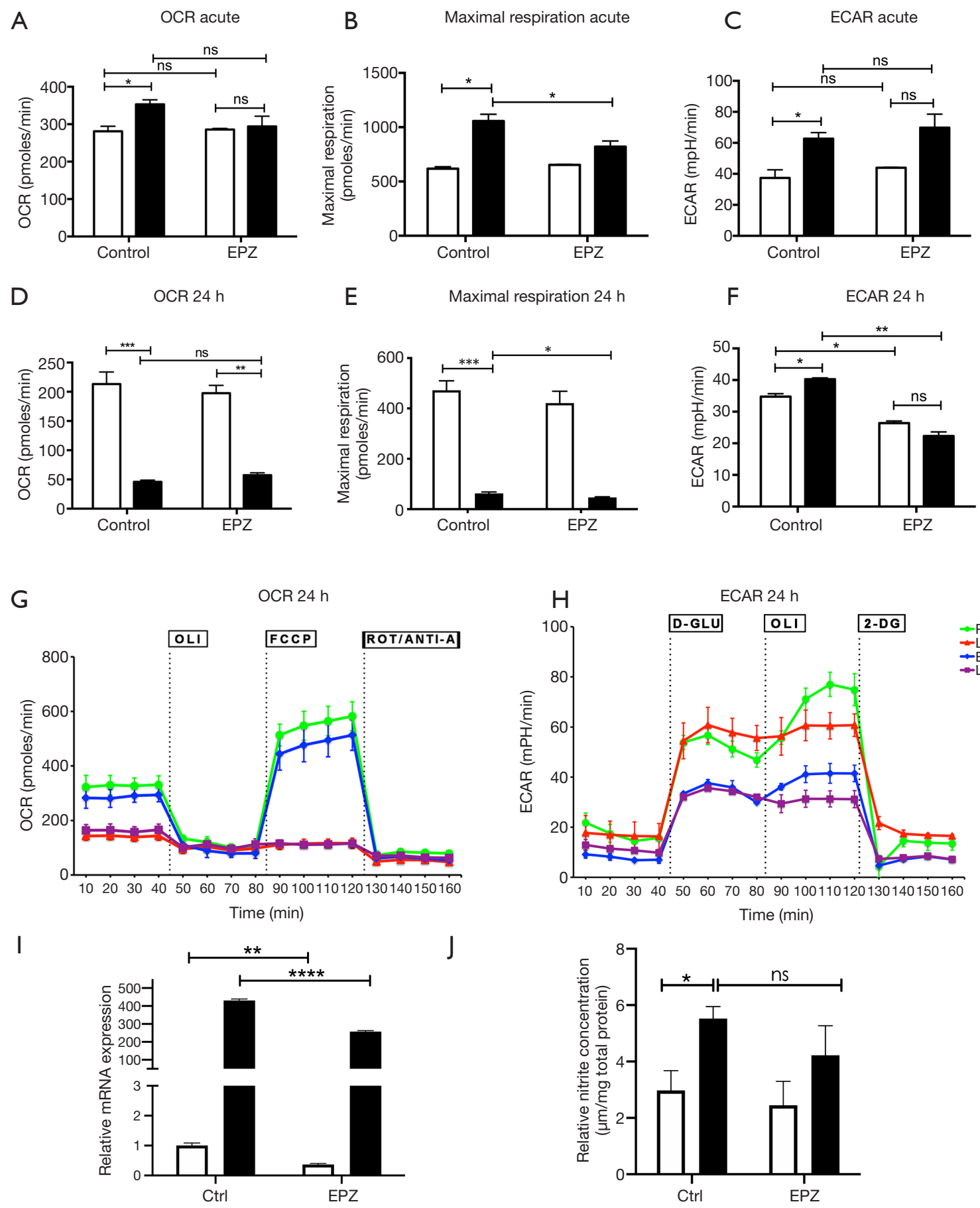

Figure 3 PRMT5 inhibition altered the DC metabolic switch. (A,B,C,D,E,F) The OCR, maximal respiration and ECAR were measured at $1 \mathrm{~h}(\mathrm{~A}, \mathrm{~B}, \mathrm{C})$ and $24 \mathrm{~h}(\mathrm{D}, \mathrm{E}, \mathrm{F})$ poststimulation. OCR and maximal respiration in response to either $2 \mu \mathrm{M}$ oligomycin or $1 \mu \mathrm{M} \mathrm{FCCP}$ were measured. The ECAR was measured before the addition of $2 \mu \mathrm{M}$ oligomycin. (G,H) Real-time changes in the OCR and ECAR were measured at $24 \mathrm{~h}$ post stimulation. (I) iNOS mRNA expression at $24 \mathrm{~h}$ poststimulation. (J) Nitrite concentration at $24 \mathrm{~h}$ poststimulation. Unpaired two-tailed Student's $t$-tests were used for data analysis in (A,B,C,D,E,F,I,J); All data are representative of 3 or more independent in vitro experiments. *, $\mathrm{P}<0.05$; ${ }^{* *}, \mathrm{P}<0.01$; ${ }^{* * *}, \mathrm{P}<0.001$; ${ }^{* * *}, \mathrm{P}<0.0001$. $\mathrm{DC}$, dendritic cell; OCR, oxygen consumption rate; ECAR, extracellular acidification rate; FCCP, Carbonyl cyanide 4-(trifluoromethoxy) phenylhydrazone; iNOS, inducible nitric oxide synthase. 
A

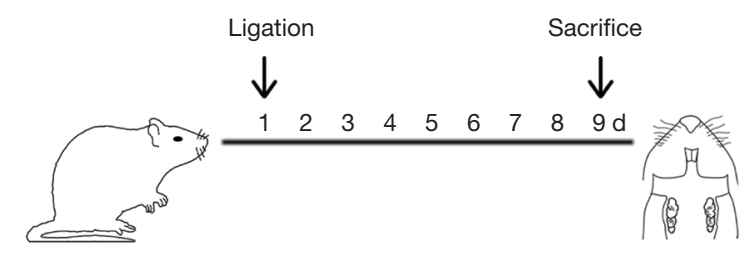

C

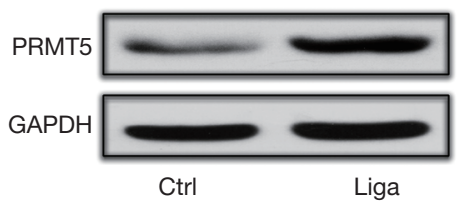

D

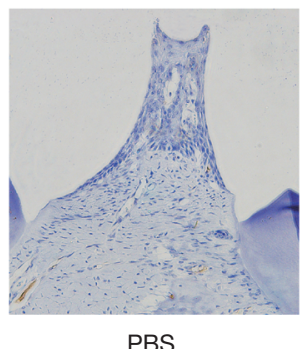

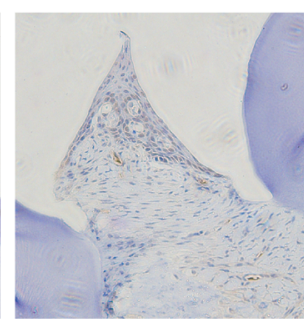

$\lg G$

B

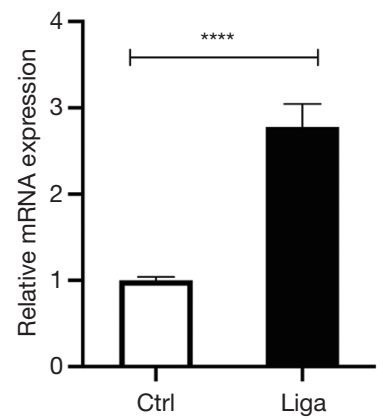

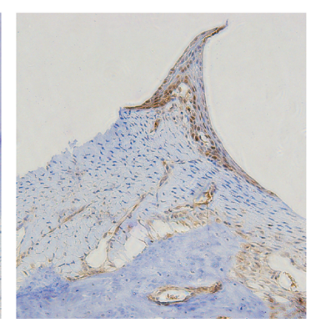

Ctrl

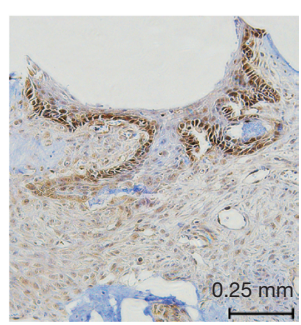

Liga

Figure 4 PRMT5 mRNA and protein expression in gingival tissues from ligatured mice and healthy mice. (A) Chart indicating the work from days 1 to 9. (B) PRMT5 mRNA expression. (C) PRMT5 protein expression from WB analysis. (D) PRMT5 protein expression from immunohistochemistry analysis. Unpaired two-tailed Student's $t$-tests were used for data analysis. ${ }^{* * *}, \mathrm{P}<0.0001$. All data are representative of 3 or more independent in vivo experiments.

mRNA upregulation induced by LPS, although the decrease in NO production was no longer obvious (Figure 3I,7).

\section{PRMT5 expression is increased in ligatured mouse gingival tissue}

To clarify the possible association between PRMT5 and periodontitis, mice weighing between 20 and $24 \mathrm{~g}$ ( 8 mice per group) in a healthy condition were administered ligatures around their second molar teeth on day 1 and sacrificed on day 9 (Figure 4A). We obtained gingival tissue from the palatal side of the ligatured teeth and isolated the mouse jaw. Gingival tissues were either subjected to total RNA extraction for assessment of mRNA expression or lysed for analysis of protein levels. Isolated maxillae were decalcified and processed for IHC staining. Compared with healthy tissue, PRMT5 mRNA was expressed at remarkably higher levels in inflamed tissue (2.8 times) (standard error: 0.266857 ) (Figure 4B). Similarly, PRMT5 protein was expressed at higher levels in inflamed tissue than in healthy tissue and was localized in both epithelial and connective tissues (Figure 4C,D).

\section{PRMT5 inbibition relieved the severity of mouse periodontitis and slowed the migration of DCs to cervical lymph nodes}

A ligature-induced mouse model was used to assess the role of PRMT5 inhibition in periodontitis beginning on day 1. PBS or EPZ015666 was injected into the palatal gingiva of the upper second molars daily from days 0 to 8 (Figure $5 A$ ). As shown in the images (10 per group), we readily observed bone loss on the palatal (1.4 times) (standard error: 0.182002) and buccal (1.7 times) (standard error: 0.301791) sides of the ligatured mice compared with the control group (palatal standard error: 0.118254/ buccal standard error: 0.093682), particularly on the buccal side (Figure 5B). Similarly, HE staining showed obvious decreases in the bone height and width in the ligatured mice (Figure 5C), and PRMT5 inhibition significantly inhibited bone loss (1.2-1.4 times) (palatal standard error: 0.121981/buccal standard error: 0.134074) (Figure 5B,C). After HE staining, we observed more inflammatory infiltration changes in ligatured mice, such as disappearance of mucosal epithelium, ulcer formation, proliferation of granulation and infiltration of inflammatory cells; however, 
A

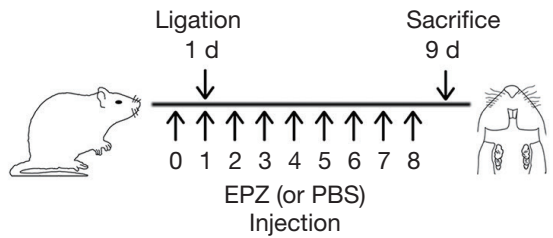

C

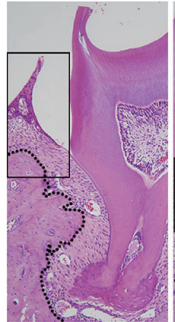

Ctrl

D

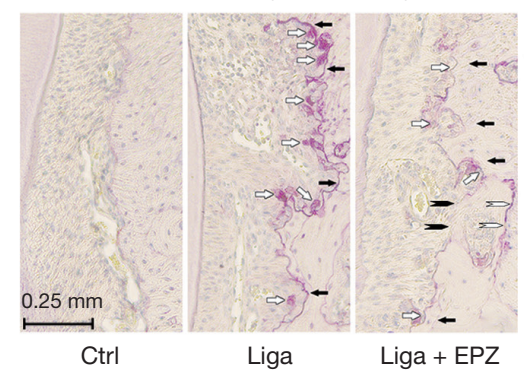

$\mathrm{F}$
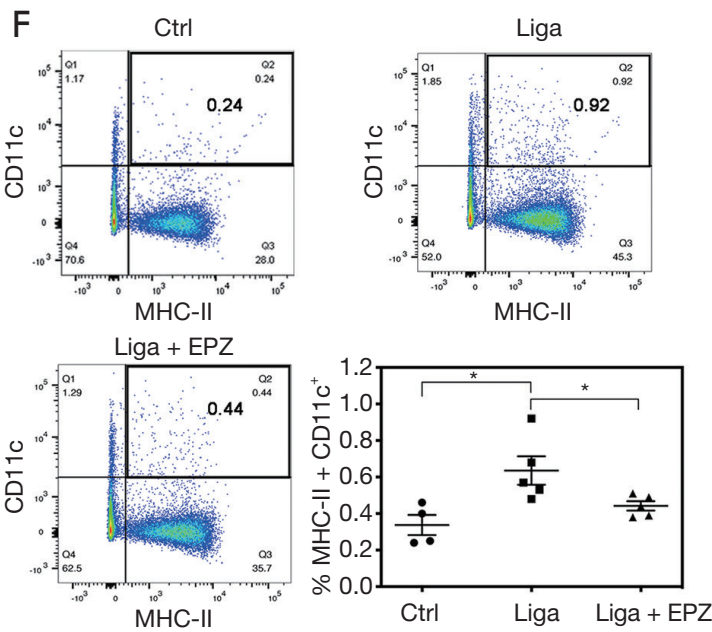

B
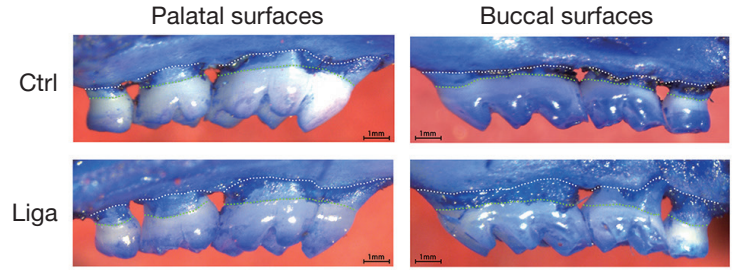

Liga + EPZ
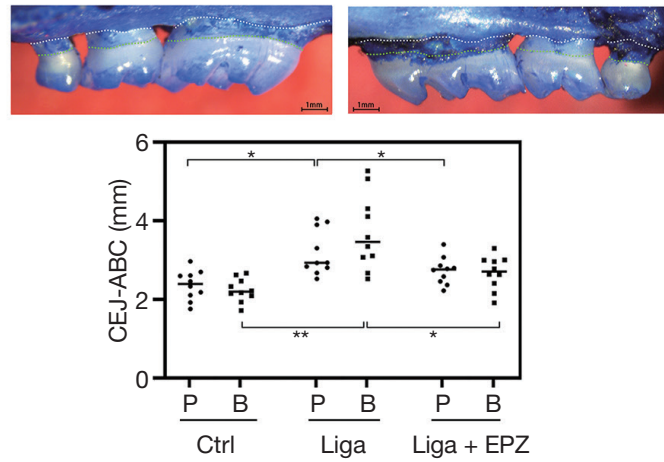

E

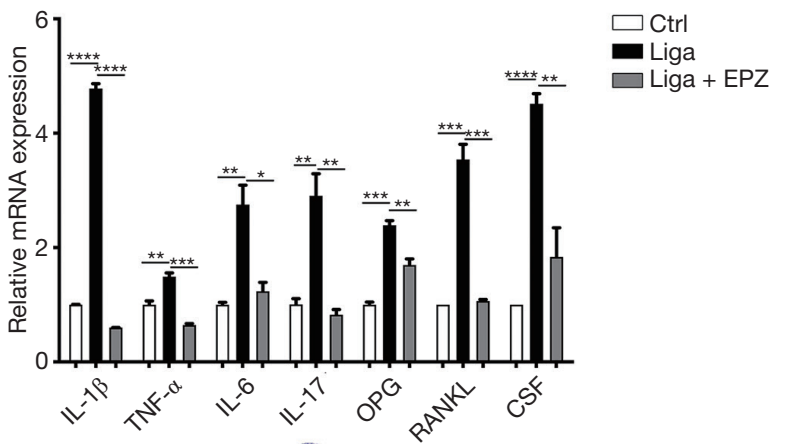

G
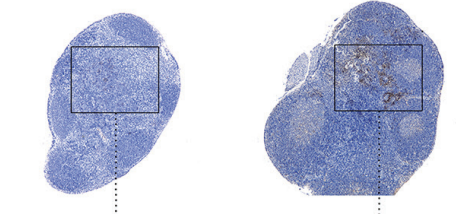

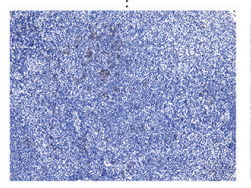

Ctrl

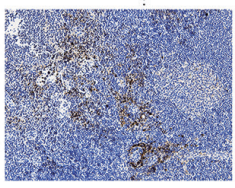

Liga
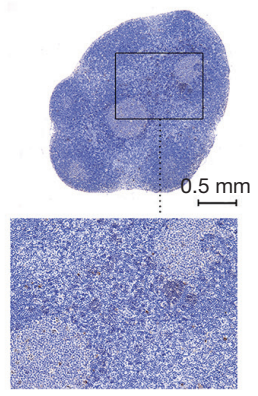

Liga + EPZ

Figure 5 The influence of PRMT5 inhibition on ligature-induced periodontitis and DC recruitment to cervical lymph nodes. (A) Chart indicating the work from days 0 to 9 . (B) Images of mouse jaws and bone loss measurements. Jaws were stained with $0.5 \%$ eosin and $1 \%$ methylene blue. The white and green dotted lines indicate the ABC and CEJ levels, respectively; bone loss was measured by comparing the distance between the ABC and CEJ. (C) HE-stained sections. Black dotted lines indicate the bone area between the $1^{\text {st }}$ and $2^{\text {nd }}$ molars. The black square indicates the area of inflammatory cell infiltration. (D) TRAP-stained sections. Small white and black arrows indicate osteoclasts and Howship's lacunae, respectively. Big white and black arrows trace the reversal line and the new bone formation. (E) RT-PCR analysis of dissected gingiva and mRNA expression of the indicated genes. (F) Flow cytometry analysis of the DC numbers in cervical lymph nodes, gated as CD11 $\mathrm{c}^{+} \mathrm{MHC}-\mathrm{II}^{+}$cells. (G) IHC analysis of CD11c protein expression in cervical lymph nodes. An unpaired two-tailed Student's $t$-test was used for data analysis. All data are representative of 4 or more independent in vivo experiments. *, $\mathrm{P}<0.05$; **, $\mathrm{P}<0.01 ;{ }^{* * *}, \mathrm{P}<0.001$; ****, $\mathrm{P}<0.0001$. DC, dendritic cell; CEJ, cementoenamel junction; ABC, alveolar bone crest; TRAP, tartrate-resistant acid phosphatase; MHC, major histocompatibility complex. 
in the PRMT5 inhibition group, only proliferation of small vessels and infiltration of a small number of inflammatory cells were observed. TRAP staining showed that PRMT5 inhibition reduced the number of osteoclasts and the formation of Howship's lacunae and increased new bone formation (Figure 5D). In addition to alveolar bone, PRMT5 inhibition also remarkably reduced the mRNA levels of proinflammatory cytokines in the gingival tissue of ligatured mice (10 per group) (1.4-1.6 times) (Figure 5E). We performed flow cytometry and IHC analyses to determine the number of DCs migrating to cervical lymph nodes after PRMT5 inhibition. The flow cytometry results (4-5 per group) showed a significant increase in the number of DCs in the cervical lymph nodes of the ligature-induced periodontitis group (1.9 times) (standard error: 0.078269) compared with the control group (standard error: 0.054829), and PRMT5 inhibition reversed the increase in the DC number (1.4 times) (standard error: 0.025962). Similar results were observed using IHC staining (Figure $5 F, G$ ).

\section{Discussion}

Impaired DC activation induces DCs to enter a tolerogenic or less active state, thereby decreasing the immune response, relieving tissue damage from excessive inflammation and attenuating immune-related diseases (36). Immature DCs (iDCs) are widespread in various tissues and function as "sentinels" by detecting pathogens. ISGs induced by IFN-I help DCs detect viruses (37). The levels of ISGs are markedly downregulated in T lymphocytes after PRMT5 inhibition (38). This inhibitory effect was observed in our study as early as $1 \mathrm{~h}$ after LPS stimulation, and PRMT5 generally inhibited all ISGs at the later time points. This tendency is consistent with an inhibitory effect of the PRMT5 inhibitor on the expression of surface costimulatory molecules and MHC molecules at 24-48 h, which are hallmarks of DC maturation $(39,40)$. Specifically, the costimulatory molecule CD40 activates DCs by interacting with $\mathrm{T}$ cells, thereby leading to the expression of CD80/ CD86, release of cytokines, and maintenance of the amplified T cell response $(41,42)$. MHC-II and MHC-I molecules bind the captured exogenous antigens and translocate them to the DC surface as a complex for $\mathrm{T}$ cell recognition $(43,44)$. Together with the decreased expression of the proinflammatory cytokines IL-12p70, TNF- $\alpha$, and IL-6 detected in our results, we observed a widespread effect of PRMT5 inhibition on DC activation and maturation after LPS stimulation. Previous studies have reported a key role for posttranslational modification of PRMT5 in promoting $\mathrm{T}$ cell function, such as $\mathrm{T}$ cell activation (45), maintenance $(22,23)$, expansion (24), and differentiation (46), and our results further support those findings by providing insights into the role of PRMT5 in DCs.

Here, for the first time, we show that PRMT5 inhibition blocks the fundamental DC metabolic switch. First, because OXPHOS serves as the major energy-producing pathway in the resting state (9), we observed a high OCR at the beginning of LPS stimulation; however, oxygen consumption decreased significantly over time. Instead, glycolytic activity increased substantially at an early time point and remained high after $24 \mathrm{~h}$, thus serving as the main energy resource to support DC activation. These findings are consistent with the results of previous studies $(7,10)$. Second, we observed a general inhibitory effect of PRMT5 on the DC metabolic switch following LPS stimulation. Although we did not observe an effect of PRMT5 inhibition on OXPHOS, PRMT5 inhibition significantly decreased the maximum mitochondrial respiration activity at both time points. The maximum mitochondrial respiration is the maximum respiration activity cells can achieve and is related to $T$ cell survival during an infection (47). This finding is consistent with the results of our previous study showing that PRMT5 inhibition decreases the expression of the prototypic Th1 cytokine IL-12p70, which is required for memory Th expansion (24). Moreover, PRMT5 inhibition showed a remarkable ability to inhibit glycolysis by itself at $24 \mathrm{~h}$ after LPS stimulation, suggesting a direct inhibitory effect of PRMT5 inhibition on the DC metabolic switch to glycolysis in the later stage, which is important for DC activation.

NO production is a possible mechanism by which the DC metabolic switch is activated $(10,48,49)$. Our results confirmed this finding because we readily detected iNOS and NO production in LPS-activated DCs. Chakhtoura (50) and Everts (10) blocked NO production by targeting related signaling pathways or administering $\mathrm{NO}$ inhibitors and observed attenuated glycolysis levels and inhibition of OXPHOS activity. However, although iNOS mRNA expression was significantly reduced in cells treated with the PRMT5 inhibitor, the inhibitor failed to block NO production to the extent we expected, which may be related to the role of arginine as an NO precursor (51). The methylation of arginine catalyzed by PRMTs may regulate NOS enzymes to reduce NO production (52); thus, PRMT5 inhibition compensates for the decrease in NOS mRNA levels by inhibiting PRMT5 activity, leading to 
increased NOS protein levels. Based on the evidence, AKT, another possible signaling pathway, regulates cell activation by modulating glycolysis through phosphorylation and activation of HK-II $(9,53)$, which is located on the mitochondrial surface and contains an AKT phosphorylation consensus sequence. Moreover, the AKT upstream kinase TREM-1 serves as an amplifier of immune signaling and the inflammatory response $(47,54)$ by binding to TLRs, after which the complex recognizes LPS and activates cells (55). The AKT downstream kinase FOXO1 also plays essential roles in DC activation and migration; for example, reduced DC function and increased susceptibility to periodontitis have been observed after FOXO1 deletion (56). Our results (Figure S1) revealed a correlation between PRMT5 inhibition and the AKT pathway in DCs, but further studies are needed to better interpret this result.

After we clarified the correlation between PRMT5 inhibition and the metabolic state of murine DCs during activation induced by LPS stimulation in vitro, we used a ligature-induced periodontitis model to assess the in vivo effect of PRMT5 inhibition. This method was first used in rodents by Rovin et al. (57). The ligature facilitates bacterial accumulation, which is the main cause of alveolar bone loss (58). This phenomenon was confirmed by several studies as described below; specifically, treatment with chlorhexidine or tetracycline hydrochloride in rats significantly relieved the severity of periodontal lesions, and similar findings were observed when mice were treated with antibiotic-containing water $(34,59,60)$. Based on these results, this model is a reliable tool to mimic human periodontitis. In our in vivo study, we observed significantly high PRMT5 mRNA and protein expression in mouse periodontitis gingival tissue, prompting us to hypothesize that PRMT5 may induce tissue destruction in this disease. This hypothesis was subsequently verified by macroscopic and histometric measurements in the mouse jaw, revealing that PRMT5 inhibition blocks alveolar bone loss and reduces osteoclast infiltration and the area of Howship's lacunae. Osteoclasts degrade the bone organic matrix by secreting acidic proteins that work together with proteinase $\mathrm{K}$. Resorption of lacunae is always accompanied by osteoclasts located on the surface, and their area and number are indicators of bone resorption (61). The effect of PRMT5 inhibition on periodontitis was further confirmed by the decreased expression of mRNAs encoding seven proinflammatory cytokines in ligatured gingiva tissue, and the destructive roles of these cytokines in periodontal inflammation have been well established in humans and animal models (62-64). After detecting and capturing bacteria, DCs become activated. At the same time, the DC morphology is reshaped, and adherens junction proteins are deregulated, leading to increased DC migration to cervical lymph nodes. Thus, treatment with a PRMT5 inhibitor impairs DC activation.

In conclusion, in the current study, we discovered that PRMT5 inhibition modulates DC activation by altering the metabolic switch, and the in vivo study indicated a promising immune-based therapeutic role of PRMT5 in periodontitis.

\section{Acknowledgments}

Funding: This work was financially supported by the National Natural Science Foundation of China (81771116), Multicenter Clinical Research Program of Shanghai Jiao Tong University School of Medicine (DLY201822) and Science and Technology Commission of Shanghai Municipality (19411950100).

\section{Footnote}

Reporting Checklist: The authors have completed the ARRIVE reporting checklist. Available at http://dx.doi. org/10.21037/atm-20-7362

Data Sharing Statement: Available at http://dx.doi. org/10.21037/atm-20-7362

Peer Review File: Available at http://dx.doi.org/10.21037/ atm-20-7362

Conflicts of Interest: All authors have completed the ICMJE uniform disclosure form (available at http://dx.doi. org/10.21037/atm-20-7362). The authors have no conflicts of interest to declare.

Ethical Statement: The authors are accountable for all aspects of the work in ensuring that questions related to the accuracy or integrity of any part of the work are appropriately investigated and resolved. All mouse experiments were approved by the Animal Care and Experiment Committee of Shanghai Jiao Tong University School of Medicine (approval ID: HKDL [2018]461), in compliance with the National institutional guidelines for the care and use of animals.

Open Access Statement: This is an Open Access article 
distributed in accordance with the Creative Commons Attribution-NonCommercial-NoDerivs 4.0 International License (CC BY-NC-ND 4.0), which permits the noncommercial replication and distribution of the article with the strict proviso that no changes or edits are made and the original work is properly cited (including links to both the formal publication through the relevant DOI and the license). See: https://creativecommons.org/licenses/by-nc-nd/4.0/.

\section{References}

1. Iwasaki A, Medzhitov R. Regulation of adaptive immunity by the innate immune system. Science 2010;327:291-5.

2. Joffre O, Nolte M A, Sporri R, et al. Inflammatory signals in dendritic cell activation and the induction of adaptive immunity. Immunol Rev 2009;227:234-47.

3. Shih VF, Davis-Turak J, Macal M, et al. Control of RelB during dendritic cell activation integrates canonical and noncanonical NF- $\kappa$ B pathways. Nat Immunol 2012;13:1162-70.

4. Bhattacharyya, S, Sen P, Wallet M, et al. Immunoregulation of dendritic cells by IL-10 is mediated through suppression of the PI3K/Akt pathway and of IB kinase activity. Blood 2004;104:1100-9.

5. Neyen C, Lemaitre B. Sensing Gram-negative bacteria: a phylogenetic perspective. Curr Opin Immunol 2016;38:8-17.

6. Jantsch J, Chakravortty D, Turza N, et al. Hypoxia and hypoxia-inducible factor-1 alpha modulate lipopolysaccharide-induced dendritic cell activation and function. J Immunol 2008;180:4697-705.

7. Krawczyk CM, Holowka T, Sun J, et al. Toll-like receptorinduced changes in glycolytic metabolism regulate dendritic cell activation. Blood 2010;115:4742-9.

8. Everts B, Amiel E, Huang S, et al. TLR-driven early glycolytic reprogramming via the kinases TBK1-IKK\& supports the anabolic demands of dendritic cell activation. Nat Immunol 2014;15:323-32.

9. Pearce EJ, Everts B. Dendritic cell metabolism. Nat Rev Immunol 2015;15:18-29.

10. Everts B, Amiel E, Windt GJ, et al. Commitment to glycolysis sustains survival of NO-producing inflammatory dendritic cells. Blood 2012;120:1422-31.

11. Roberts DJ, Miyamoto S. Hexokinase II integrates energy metabolism and cellular protection: Akting on mitochondria and TORCing to autophagy. Cell Death Differ 2015;22:248-57.

12. Ohtani M, Nagai S, Kondo S, et al. Mammalian target of rapamycin and glycogen synthase kinase 3 differentially regulate lipopolysaccharide-induced interleukin-12 production in dendritic cells. Blood 2008;112:635-43.

13. Yang Y, Hadjikyriacou A, Xia Z, et al. PRMT9 is a type II methyltransferase that methylates the splicing factor SAP145. Nat Commun 2015;6:6428.

14. Pal S, Baiocchi RA, Byrd JC, et al. Low levels of miR-92b/96 induce PRMT5 translation and H3R8/ H4R3 methylation in mantle cell lymphoma. EMBO J 2007;26:3558-69.

15. Wang L, Pal S, Sif S. Protein arginine methyltransferase 5 suppresses the transcription of the RB family of tumor suppressors in leukemia and lymphoma cells. Mol Cell Biol 2008;28:6262-77.

16. Powers MA, Fay MM, Factor RE, et al. Protein arginine methyltransferase 5 accelerates tumor growth by arginine methylation of the tumor suppressor programmed cell death 4. Cancer Res 2011;71:5579-87.

17. Cho EC, Zheng S, Munro S, et al. Arginine methylation controls growth regulation by E2F-1. EMBO J 2012;31:1785-97.

18. Kim JH, Yoo BC, Yang WS, et al. The Role of Protein Arginine Methyltransferases in Inflammatory Responses. Mediators Inflamm 2016;2016:4028353

19. Krzystek-Korpacka M, Fleszar MG, Bednarz-Misa I, et al. Transcriptional and Metabolomic Analysis of L-Arginine/Nitric Oxide Pathway in Inflammatory Bowel Disease and Its Association with Local Inflammatory and Angiogenic Response: Preliminary Findings. Int J Mol Sci 2020;21:1641.

20. Chen D, Zeng S, Huang M, et al. Role of protein arginine methyltransferase 5 in inflammation and migration of fibroblast-like synoviocytes in rheumatoid arthritis. J Cell Mol Med 2017;21:781-90.

21. Bandyopadhyay S, Harris DP, Adams GN, et al. HOXA9 methylation by PRMT5 is essential for endothelial cell expression of leukocyte adhesion molecules. Mol Cell Biol 2012;32:1202-13.

22. Inoue $\mathrm{M}$, Okamoto $\mathrm{K}$, Terashima A, et al. Arginine methylation controls the strength of $\gamma c$-family cytokine signaling in T cell maintenance. Nat Immunol 2018;19:1265-76.

23. Snyder KJ, Zitzer NC, Gao Y, et al. PRMT5 regulates $\mathrm{T}$ cell interferon response and is a target for acute graftversus-host disease. JCI Insight 2020;5:e131099.

24. Webb LM, Amici SA, Jablonski KA, et al. PRMT5Selective Inhibitors Suppress Inflammatory $\mathrm{T}$ Cell Responses and Experimental Autoimmune 
Encephalomyelitis. J Immunol 2017;198:1439-51.

25. Webb LM, Sengupta S, Edell C, et al. Protein Arginine Methyltransferase 5 Promotes Cholesterol BiosynthesisMediated Th17 Responses and Autoimmunity. J Clin Invest 2020;130:1683-98.

26. Ding Z, Li G, Deng Z, et al. Circ-PRMT5 Enhances the Proliferation, Migration and Glycolysis of Hepatoma Cells by Targeting miR-188-5p/HK2 Axis. Ann Hepatol 2020;19:269-79.

27. Tonetti MS, Claffey N. Advances in the progression of periodontitis and proposal of definitions of a periodontitis case and disease progression for use in risk factor research. Group C consensus report of the 5th European workshop in Periodontology. J Clin Periodontol 2005;32:210-3.

28. Banchereau J, Briere F, Caux C, et al. Immunobiology of dendritic cells. Annu Rev Immunol 2000;18:767-811.

29. Chan-Penebre E, Kuplast KG, Majer CR, et al. A selective inhibitor of PRMT5 with in vivo and in vitro potency in MCL models. Nat Chem Biol 2015;11:432-7.

30. Zheng BN, Ding CH, Chen SJ, et al. Targeting PRMT5 Activity Inhibits the Malignancy of Hepatocellular Carcinoma by Promoting the Transcription of HNF4alpha. Theranostics 2019;9: 2606-17.

31. Nagai Y, Ji MQ, Zhu F, et al. PRMT5 Associates With the FOXP3 Homomer and When Disabled Enhances Targeted p185erbB2/neu Tumor Immunotherapy. Front Immunol 2019;10:174.

32. Baker PJ, Dixon M, Roopenian DC. Genetic control of susceptibility to Porphyromonas gingivalis-induced alveolar bone loss in mice. Infect Immun 2000;68:5864-8.

33. Madaan A, Verma R, Singh AT, et al. A stepwise procedure for isolation of murine bone marrow and generation of dendritic cells. J Biol Methods 2014;1:e1.

34. Abe T, Hajishengallis G. Optimization of the ligatureinduced periodontitis model in mice. $\mathrm{J}$ Immunol Methods 2013;394:49-54.

35. Mizraji G, Segev H, Wilensky A, et al. Isolation, Processing and Analysis of Murine Gingival Cells. J Vis Exp 2013;77:e50388.

36. Cools N, Ponsaerts P, Tendeloo V, et al. Balancing Between Immunity and Tolerance: An Interplay Between Dendritic Cells, Regulatory T Cells, and Effector T Cells. J Leukoc Biol 2007;82:1365-74.

37. Schulte BM, Kers-Rebel ED, Prosser AC, et al. Differential susceptibility and response of primary human myeloid bdca1+ dendritic cells to infection with different enteroviruses. PLoS One 2013;8:e62502.

38. Metz PJ, Ching KA, Xie T, et al. Symmetric Arginine
Dimethylation Is Selectively Required for mRNA Splicing and the Initiation of Type I and Type III Interferon Signaling. Cell Rep 2020;30:1935-1950.e8.

39. Chang SK, Mihalcik SA, Jelinek DF. B lymphocyte stimulator regulates adaptive immune responses by directly promoting dendritic cell maturation. J Immunol 2008;180:7394-403.

40. Sacramento L, Trevelin SC, Nascimento MS, et al. Toll-Like Receptor 9 Signaling in Dendritic Cells Regulates Neutrophil Recruitment to Inflammatory Foci following Leishmania infantum Infection. Infect Immun 2015;83:4604-16.

41. Mangsbo SM, Broos S, Fletcher E, et al. The Human Agonistic CD40 Antibody ADC-1013 Eradicates Bladder Tumors and Generates T-cell-dependent Tumor Immunity. Clin Cancer Res 2015;21:1115-26.

42. Vitale LA, Thomas LJ, He LZ, et al. Development of CDX-1140, an agonist CD40 antibody for cancer immunotherapy. Cancer Immunol Immunother 2019;68:233-45.

43. Yan J, Liu B, Shi Y, et al. Class II MHC-independent suppressive adhesion of dendritic cells by regulatory $\mathrm{T}$ cells in vivo. J Exp Med 2017;214:319-26.

44. Sato K, Uto T, Fukaya T, et al. Regulatory Dendritic Cells. Curr Top Microbiol Immunol 2017;410:47-71.

45. Blanchet F, Cardona A, Letimier F, et al. CD28 costimulatory signal induces protein arginine methylation in T cells. J Exp Med 2005;202:371-7.

46. Geoghegan V, Guo A, Trudgian D, et al. Comprehensive Identification of Arginine Methylation in Primary T Cells Reveals Regulatory Roles in Cell Signalling. Nat Commun 2015;6:6758.

47. Duan M, Wang ZC, Wang XY, et al. TREM-1, an inflammatory modulator, is expressed in hepatocellular carcinoma cells and significantly promotes tumor progression. Ann Surg Oncol 2015;22:3121-9.

48. Almeida A, Moncada S, Bolanos JP. Nitric oxide switches on glycolysis through the AMP protein kinase and 6-phosphofructo-2-kinase pathway. Nat Cell Biol 2004;6:45-51.

49. Amiel E, Everts B, Fritz D, et al. Mechanistic target of rapamycin inhibition extends cellular lifespan in dendritic cells by preserving mitochondrial function. J Immunol 2014;193:2821-30.

50. Chakhtoura M, Chain RW, Sato PY, et al. Ethyl pyruvate modulates murine dendritic cell activation and survival through their immunometabolism. Front Immunol 2019;10:30. 
51. Sureda A, Pons A. Arginine and Citrulline Supplementation in Sports and Exercise: Ergogenic Nutrients? Med Sport Sci 2012;59:18-28.

52. Redel BK, Tessanne KJ, Spate LD, et al. Arginine increases development of in vitro produced porcine embryos and affects the protein arginine methyltransferasedimethylarginine dimethylaminohydrolase-nitric oxide axis. Reprod Fertil Dev 2015;27:655-66.

53. John S, Weiss JN, Ribalet B. Subcellular localization of hexokinases I and II directs the metabolic fate of glucose. PLoS One 2011;6:e17674.

54. Baruah S, Murthy S, Keck K, et al. TREM-1 regulates neutrophil chemotaxis by promoting NOX-dependent superoxide production. J Leukoc Biol 2019;105:1195-207.

55. Fortin CF, Lesur O, Fulop T Jr. Effects of TREM-1 activation in human neutrophils: activation of signaling pathways, recruitment into lipid rafts and association with TLR4. Int Immunol 2007;19:41-50.

56. Xiao W, Dong G, Pacios S, et al. FOXO1 deletion reduces dendritic cell function and enhances susceptibility to periodontitis. Am J Pathol 2015;185:1085-93.

57. Rovin S, Costich ER, Gordon HA. The influence of bacteria and irritation in the initiation of periodontal disease in germfree and conventional rats. J Periodontal Res 1966;1:193-204.

Cite this article as: Mi W, Qiao S, Zhang X, Wu D, Zhou L, Lai H. PRMT5 inhibition modulates murine dendritic cells activation by inhibiting the metabolism switch: a new therapeutic target in periodontitis. Ann Transl Med 2021;9(9):755. doi: 10.21037/atm-20-7362
58. Graves DT, Fine D, Teng YT, et al. The use of rodent models to investigate host-bacteria interactions related to periodontal diseases. J Clin Periodontol 2008;35:89-105.

59. Kenworthy R, Baverel M. Studies of a periodontal tissue lesion in the rat, untreated or treated with chlorhexidine digluconate. J Clin Periodontol 1981;8:349-58.

60. Weiner GS, DeMarco TJ, Bissada NF. Long term effect of systemic tetracycline administration on the severity of induced periodontitis in the rat. J Periodontol 1979;50:619-23.

61. Kylmaoja E, Nakamura M, Tuukkanen J. Osteoclasts and Remodeling Based Bone Formation. Curr Stem Cell Res Ther 2016;11:626-33.

62. Vernal R, Dutzan N, Chaparro A, et al. Levels of interleukin-17 in gingival crevicular fluid and in supernatants of cellular cultures of gingival tissue from patients with chronic periodontitis. J Clin Periodontol 2005;32:383-9.

63. Gaffen SL, Hajishengallis G. A new inflammatory cytokine on the block: re-thinking periodontal disease and the Th1/ Th2 paradigm in the context of Th17 cells and IL-17. J Dent Res 2008;87:817-28.

64. Garlet GP. Destructive and protective roles of cytokines in periodontitis: A re-appraisal from host defense and tissue destruction viewpoints. J Dent Res 2010;89:1349-63. 


\section{Supplementary}

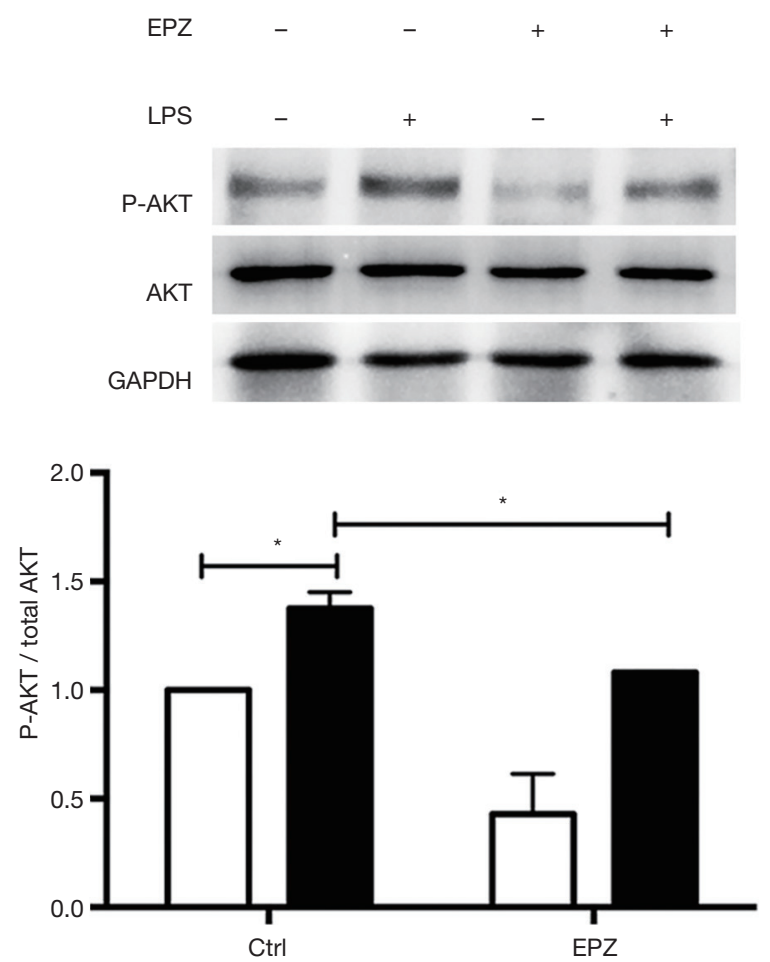

Figure S1 AKT phosphorylation at $24 \mathrm{~h}$ poststimulation. The densitometry results are shown as the ratio of p-AKT to total AKT. The paired ratio two-tailed Student's $t$-test were used for the data analysis. *, $\mathrm{P}<0.05$. 